\title{
Potenziale und Umsetzung der Digitalisierung auf Unternehmensebene
}

\author{
Digitalisierung auf Unternehmensebene ist längst praktischer Bestandteil strategischer \\ Planungen und Umsetzungen. Dabei lassen sich spezifische Auswirkungen auf das \\ Produktivitätswachstum oder die Beschäftigung anhand von Produzenten-Kunden- \\ Beziehungen aufzeigen. Mit Blick auf das Produktivitätswachstum ist zu erwarten, dass \\ Steigerungen nur verzögert auftreten und in vielen Bereichen noch unausgeschöpfte Potenziale \\ liegen. Positive Beschäftigungseffekte sind insbesondere im Business-to-Business-Markt \\ zu erwarten, der Business-to-Consumer-Markt, der von den großen Tech-Unternehmen \\ dominiert wird, weist vergleichsweise geringe Beschäftigtenzahlen auf. Unterm Strich wird \\ es in zunehmendem Maße zu einer Veränderung der Kompetenzanforderungen kommen. Die \\ Herausforderung besteht darin, weniger innovationsaffine Unternehmen und weniger IT-affine \\ Beschäftigte nicht abzuhängen.
}

\begin{abstract}
Der Begriff der Digitalisierung ist seit Jahren in aller Munde und auch auf Unternehmensebene kommt kaum noch ein Strategiepapier heraus, das nicht diesen Megatrend als zentrale Zukunftsherausforderung benennt und adressiert. Der Vielschichtigkeit der Veränderungen, denen Unternehmen beim Übergang aus einer analogen in eine digitale Welt gegenüberstehen, steht dabei häufig ein hohes $M a ß$ an Unschärfe der Begrifflichkeit gegenüber, die zu einer Vermischung der vielen Konzepte und Ideen rund um die Digitalisierung beiträgt. Im deutschsprachigen Raum liegt dies insbesondere daran, dass sich hier - im Gegensatz zur englischen Sprache - noch keine differenzierten Begriffe herausgebildet haben, die eine gewisse Struktur vorgeben.
\end{abstract}

So beschreibt etwa "Digitisation“ im Englischen eine Digitalisierung im fundamentalen, basalen Sinne, also die Umwandlung eines vormals analogen Prozesses in eine digitale Form. Dies geschieht z. B., wenn ein Unternehmen ein ana-

(C) Der/die Autor(en) 2020. Open Access: Dieser Artikel wird unter der Creative Commons Namensnennung 4.0 International Lizenz (http:// creativecommons.org/licenses/by/4.0/deed.de) veröffentlicht.

Open Access wird durch die ZBW - Leibniz-Informationszentrum Wirtschaft gefördert.

Prof. Dr. Michael Hüther ist Direktor des Instituts der deutschen Wirtschaft (IW) in Köln und Hochschullehrer an der European Business School in OestrichWinkel. loges Archiv ehemals handschriftlicher Korrespondenzen in ein digitales, etwa E-Mail-basiertes System überträgt. Wesentlich tiefgreifender ist demgegenüber die „Digitalisation“. Diese umfasst den Wandel vormals analoger Geschäftsmodelle in digitale Pendants: Wo Kunden früher persönlich in einem Geschäft Produkte ausgewählt und gekauft haben, besteht heute mit einem entsprechenden Endgerät ortsungebunden die Kaufmöglichkeit über digitale Plattformen wie beispielsweise Amazon oder Alibaba. Die Veränderungen durch Digitalisation betreffen also die Geschäftsmodelle und setzen dabei eine Digitisation der Prozesse bis zu einem gewissen Punkt voraus. Den Grad einer „Digital Transformation" erhält Digitalisierung erst, wenn Software-basierte Prozesse und Algorithmen dazu beitragen, dass sich die Agilität von Unternehmen verbessert und die Chance genutzt wird, in Echtzeit auf veränderte Marktumstände und Kundenwünsche reagieren zu können. Während Digitalisation im Unternehmen auch innerhalb einzelner Siloprojekte durchgeführt werden kann, geht Digital Transformation darüber hinaus und bezeichnet eine Nachfrage-basierte, strategische Anpassung des gesamten Unternehmens. ${ }^{1}$

Digitalisierung kann auf Ebene der Prozesse, der Produkte sowie der Geschäftsmodelle betrachtet werden. Prozesse

1 Vgl. O. Bendel: Digitalisierung, in: Gabler Wirtschaftslexikon, Wiesbaden, https://wirtschaftslexikon.gabler.de/definition/digitalisierung54195 (16.12.2019); Centery: Digitization, Digitalization and Digital Transformation, https://performance.centerity.com/digitization-digitalization-and-digital-transformation (27.2.2020); J. Bloomberg: Digitization, Digitalization, And Digital Transformation: Confuse Them At Your Peril, 29.4.2018, https://www.forbes.com/sites/jasonbloomberg/2018/04/29/digitization-digitalization-and-digital-transformation-confuse-them-at-your-peril/ (27.2.2020). 
Abbildung 1

Geschäftsmodelle der digitalen Transformation in

Produzenten-Kunden-Beziehungen

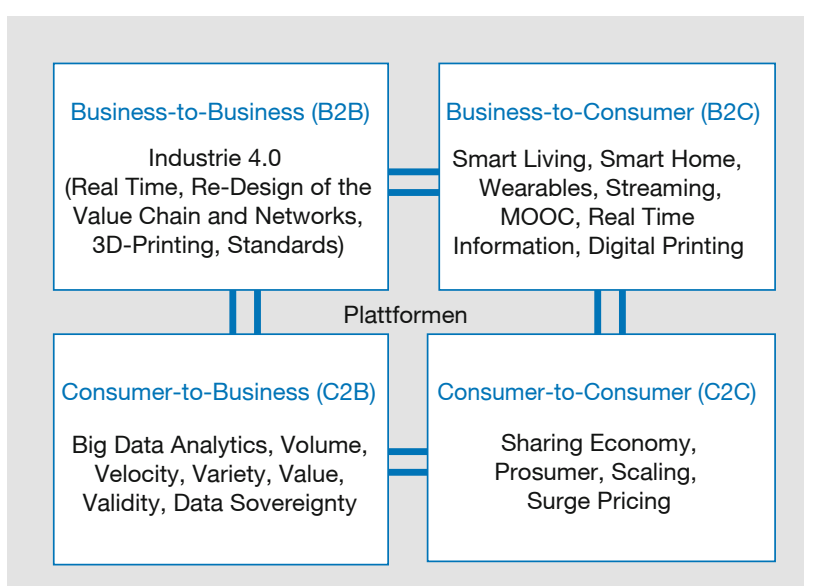

Quelle: Digitalisierung: Motor im Strukturwandel - Herausforderung für die Wirtschaftspolitik, in: ORDO, Jahrbuch für die Ordnung von Wirtschaft und Gesellschaft, 68. Jg. (2018), H. 1, S. 187.

und Produkte haben einerseits im Zuge der digitalen Technologien das Potenzial, sich in ihrer Qualität zu verbessern, andererseits entstehen auch gänzlich neue Geschäftsmodelle.

\section{Eine Kategorisierung nach Konsumenten- Unternehmen-Schnittstellen}

Die Unterteilung nach Digitisation, Digitalisation und Digital Transformation ist als Präzisierung des Begriffs der „Digitalisierung" zwar hilfreich, um etwa zwischen Prozessen oder Geschäftsmodellen zu unterscheiden, für eine tiefergehende Analyse beispielsweise der Produktivitäts- und Arbeitsmarkteffekte ist jedoch eine weitergehende Spezifikation nach Wirkungskontext notwendig. Aufgrund der fundamentalen Unterschiede zwischen Aspekten der Digitalisation innerhalb der industriellen Wertschöpfungsketten sowie im Markt für Konsumgüter bietet sich hierfür eine Kategorisierung nach Produzenten-Kunden-Beziehung an. Betrachtet man Unternehmen und Konsumenten als Hauptakteure, spannen sich damit in der digitalen Welt vier Schnittstellen mit völlig unterschiedlichen Geschäftsmodellen auf:

1. Business-to-Business (B2B),

2. Business-to-Consumer (B2C),

3. Consumer-to-Business (C2B) und

4. Consumer-to-Consumer (C2C) (vgl. Abbildung 1). ${ }^{2}$

2 Der folgende Abschnitt basiert auf M. Hüther: Digitalisierung: Motor im Strukturwandel - Herausforderung für die Wirtschaftspolitik, in: ORDO, Jahrbuch für die Ordnung von Wirtschaft und Gesellschaft, 68. Jg. (2018), H. 1, S. 179-216.
Abbildung 2

Verbundwertschöpfung im internationalen Vergleich Wertschöpfungsanteil an der Gesamtwirtschaft

in \%, 2014

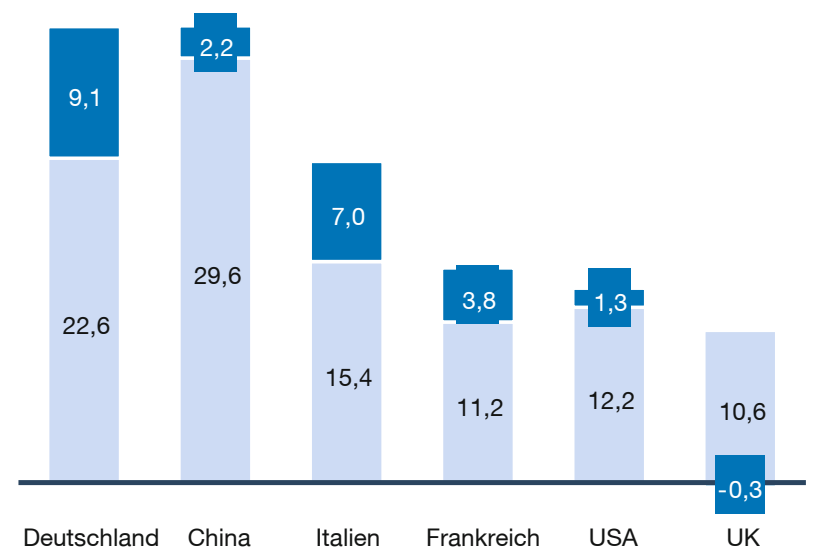

Wertschöpfung Verarbeitendes Gewerbe घerbundwertschöpfung Quelle: OECD (2018), Institut der deutschen Wirtschaft.

Für die Digitalisierung der B2B-Schnittstelle steht das Konzept Industrie 4.0 im Vordergrund, mit dem die Umsetzung digitaler Kommunikationstechnologien zwischen Maschinen beschrieben wird und die sich im Idealfall über die gesamte Wertschöpfungskette erstreckt. Da sich nirgends sonst in Europa innerhalb von hochspezialisierten Clustern ein so verzahntes industrielles Ökosystem entwickelt hat, besteht für die Digitalisierung der Industrieproduktion in Deutschland ein besonders großes Potenzial. ${ }^{3} \mathrm{Im}$ Vordergrund steht hier einerseits die Verbesserung der Prozesse durch automatisierte Machine-to-MachineKommunikation (Digitisation), andererseits entstehen durch die Einbindung dienstleistungsbezogener Leistungen in Industrieprodukte neue Geschäftsmodelle (Digitalisation). Letzteres ist ein Alleinstellungsmerkmal der deutschen Industrie, das sich in einem im internationalen Vergleich bemerkenswert hohen Anteil der sogenannten Verbundwertschöpfung - dem Anteil an komplementären industrienahen Dienstleistungen - spiegelt (vgl. Abbildung 2). Schon jetzt reüssieren deutsche Unternehmen dadurch, dass sie innovative Angebote kreieren, die sich über spezielle Dienstleistungen kundenspezifisch differenzieren lassen. Eine Individualisierung des Angebots bis hin zur Losgröße 1 ohne übermäßigen Steuerungsaufwand und zu Bedingungen der Kostenführerschaft - also der Aufbruch in die Digital Transformation - ist eine der

3 Vgl. C. Ketels, S. Protsiv: European Cluster Panorama 2014, in: European Commission (Hrsg.): European Cluster Observatory Report, Center for Strategy and Competitiveness, Stockholm School of Economics, 2014. 
Verheißungen, mit denen die industrielle Digitalisierung aufwarten kann. ${ }^{4}$

Ganz anders zeigt die Digitalisierung inr Gesicht in der Business-to-Customer-Schnittstelle in Form der großen Tech-Unternehmen des Silicon Valleys - zunehmend auch in Form entsprechender Pendants aus China. Die Konzerne haben die Digital Transformation wahrlich erfolgreich umgesetzt und werden in der öffentlichen Wahrnehmung mit ihren skalierbaren, weil grundsätzlich orts- und kulturungebundenen - und bei regionalen Spezifika durchaus differenzierbaren - Dienstleistungen wohl am ehesten als Gesichter des digitalen Wandels wahrgenommen. Den Konsumenten werden über riesige Onlineplattformen große Netzwerkeffekte geboten, bei deren Realisierung sie Bewertungs-, Such- und Koordinationskosten sparen.

Die Plattformen besetzen zwar wichtige Knotenpunkte der B2C-Welt, andere Akteure können aber über die horizontale Integration neuer Produkte durchaus an diesem Erfolg teilhaben - die sogenannte „inverse Skalierbarkeit". Google Maps ist etwa ein klassisches B2C-Produkt, das wiederum Potenziale für "nachgelagerte“ Geschäftsmodelle wie verschiedene Google-Maps-basierte Mobilitätsangebote bietet. Die großen Internetkonzerne öffnen damit ein latentes Innovationspotenzial und bleiben gewissermaßen am Ende ihrer Wertschöpfungskette „angreifbar". Aktuell ist noch völlig unklar, an welcher Stelle in der Wertschöpfungskette es Unternehmen in Zukunft schaffen werden, Kundenbedürfnisse sowie Kundendaten zu monetarisieren.

Digitalisierung in der C2B-Schnittstelle steht dem spiegelbildlich gegenüber und hat ihre besondere Ausprägung im Bereich Big Data ${ }^{5}$. Unternehmen „kaufen“ Daten von Kunden und bezahlen mit Dienstleistungen. Dabei ist dieser "Datenverkauf“ mitunter unfreiwillig, unbewusst oder zumindest unreflektiert. Unternehmen benutzen diese Daten wiederum, um neue Geschäftsfelder zu bespielen (Digitalisation) oder Prozesse algorithmengesteuert in Echtzeit anzupassen (Digital Transformation). Wie keine andere Schnittstelle ist der C2B-Markt vom Trade-off zwischen ständiger Qualitätssteigerung und „ressour-

4 Vgl. IW Consult, Institut der deutschen Wirtschaft: Industry as a growth engine in the global economy, Köln 1.12.2013, https://www. businesseurope.eu/sites/buseur/files/media/imported/201400243-E.pdf (16.12.2019); McKinsey Global Institute: Making it in America: Revitalizing US Manufacturing, 2017, S. 28 ff., https://www. mckinsey.com/ /media/mckinsey/featured\%20insights/Americas/ Making\%20it\%20in\%20America\%20Revitalizing\%20US\%20manufacturing/Making-it-in-America-Revitalizing-US-manufacturing-Fullreport.ashx (15.12.2019).

5 Vgl. B. Engels, H. Goecke: Big Data in Wirtschaft und Wissenschaft, IW-Analyse, Nr. 130, Köln 2019, https://www.iwkoeln.de/studien/iwanalysen/beitrag/barbara-engels-henry-goecke-big-data-in-wirtschaft-und-wissenschaft.html (7.2.2020). cenneutraler" Verbesserung der Wertschöpfung auf der einen Seite sowie einem Eingriff in die Privatsphäre auf der anderen Seite geprägt.

Wiederum anders zeigt sich die Digitalisierung in der C2C-Schnittstelle hauptsächlich im Erstarken der Sharing Economy, in der jeder Konsument zu einem potenziellen Anbieter von Gütern oder Dienstleistungen wird - von Autos über Wohnungen bis hin zu Direktfinanzierungen am Kapitalmarkt. Der C2C-Markt ist in besonderer Weise abhängig von Plattformen, die als digitaler Intermediär Anbieter und Nachfrager zusammenbringen und das nötige Vertrauen schaffen. Ermöglicht wird der Erfolg von C2C-Lösungen durch gesunkene Vernetzungskosten: Denn wenn Transaktionskosten aufgrund der schnellen digitalen Vernetzung gegen Null gehen, liegen auch die Grenzkosten der Verbreitung einer zusätzlichen Information innerhalb eines bestehenden Systems bei nahe null.

Die Beschreibung der unterschiedlichen Schnittstellen deutet bereits an, dass insbesondere mit Blick auf Produktivität und Beschäftigung signifikante Unterschiede bestehen könnten. Die folgende Analyse hat zum Ziel, diese konsistent herauszuarbeiten. Dafür ist bereits ein Hinweis in den vier Schnittstellen gegeben: Denn lediglich im B2B-Bereich ist originär den Unternehmen die Aufgabe gestellt, aus ihren etablierten Prozessketten nutzbare Datenbestände zu machen. Dass dies eine besondere Herausforderung ist, die zudem enorme Ressourcen für das Datenmanagement benötigt, wird immer offenkundiger. ${ }^{6}$ An den anderen Schnittstellen ist dies insofern anders, als die digitalen Geschäftsmodelle uno actu mit Digitisation sowie Digitalisation verbunden sind.

\section{Die große digitale Verheißung:}

Produktivitätswachstum

Positive gesamtwirtschaftliche Effekte werden im Zuge des digitalen Wandels insbesondere von der Produktivitätsentwicklung erwartet. Renommierte Forscher und Internetpioniere wie Brynjolfsson und $\mathrm{McAfee}^{7}$ sowie Mokyr ${ }^{8}$ haben bereits vor einigen Jahren einen Produktivitätsschub vorhergesagt. Dass dieser in Deutschland wie übrigens auch in anderen Industrienationen - bislang ausgeblieben ist, zeigt sich deutlich in Abbildung 3 . So hat sich der Beitrag der totalen Faktorproduktivität zum Produktionspotenzial seit 2005 lediglich auf dem nied-

6 Vgl. DEMAND Projekt, https://www.demand-projekt.de/ (30.1.2020)

7 Vgl. E. Brynjolfsson, A. McAfee: The Second Machine Age. Work, Progress, and Prosperity in a Time of Brilliant Technologies, New York 2014.

8 Vgl. J. Mokyr: The Next Age of Invention: Technology's Future Is Brighter Than Pessimists Allow, in: City Journal, Winter (2014), S. 1220. 
Abbildung 3

Schätzungen des preisbereinigten

Produktionspotenzials der deutschen Wirtschaft

in \%, Beiträge zum Produktionspotenzial in Prozentpunkten

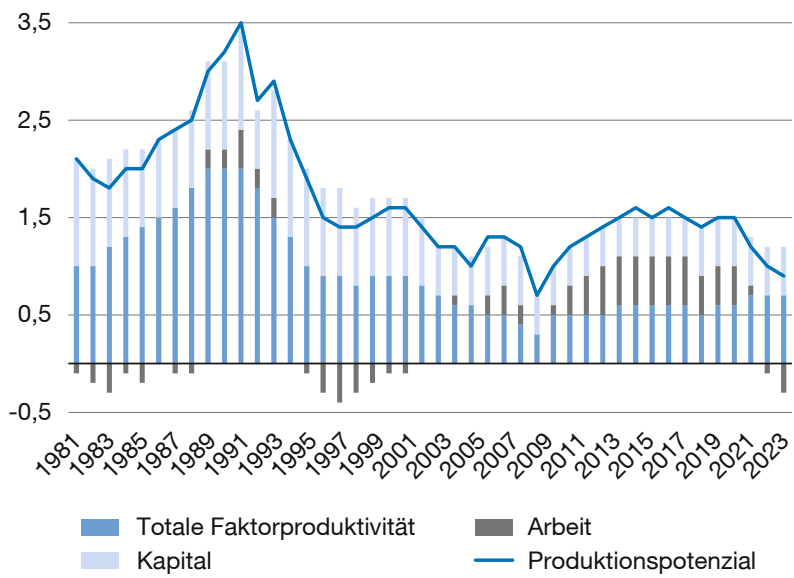

Quelle: BMWI Herbstprojektion 2019, Institut der deutschen Wirtschaft.

rigen Niveau von leicht über 0,5 Prozentpunkten stabilisiert. ${ }^{9}$ Der auf Makroebene nicht vorhandene Zusammenhang zwischen voranschreitendem digitalen Wandel und der Produktivitätsentwicklung wird mitunter als Produktivitätsparadoxon der Digitalisierung bezeichnet.

Häufig wird in diesem Kontext prominent eingebracht, dass Produktivitätseffekte in großen Umbruchphasen deutlich verzögert eintreten und auch die aktuell zu beobachtenden Veränderungen aufgrund von hohen Investitions- und Umstellungskosten bislang noch kaum sichtbar geworden sind, ein Produktivitätsschub in Zukunft hingegen durchaus zu erwarten ist. ${ }^{10}$ Dennoch erscheint es plausibel, dass in den unterschiedlichen Schnittstellen auch unterschiedliche digitalisierungsbedingte Produktivitätseffekte erzielt werden, die auf gesamtwirtschaftlicher Ebene ein gemischtes Bild abgeben.

9 Die totale Faktorproduktivität (TFP) bemisst als Residualgröße unabhängig vom Einsatz der Produktionsfaktoren das Produktivitätswachstum, das durch technischen und organisatorischen Fortschritt zustande kommt.

10 Als Analogie dient die Einführung der Elektrizität, die zwar schon Ende der 1890er Jahre in amerikanischen Fabriken verwendet wurde, aber erst über 20 Jahre später zu nennenswerten Produktivitätsgewinnen führte. Vgl. B. van Ark et al.: Navigating the New Digital Economy: Driving Digital Growth and Productivity from Installation to Deployment, 2016, https://www.conference-board.org/publications/publicationdetail.cfm? publicationid=7215\&topicid=10\&subtopicid $=70$. (17.12.2019)
Abbildung 4

Digitalisierung als Treiber von Produktivität?

2018

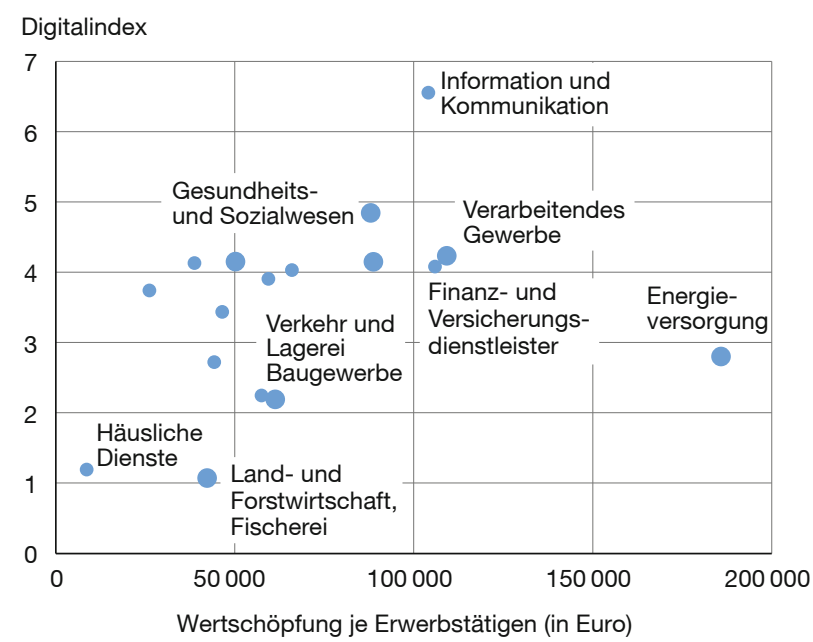

Quellen: IW Consult: Digital-Atlas Deutschland: Überblick über die Digitalisierung von Wirtschaft und Gesellschaft sowie von KMU, NGOs, Bildungseinrichtungen sowie der Zukunft der Arbeit in Deutschland, Köln 2018, https://www.iwkoeln.de/fileadmin/user_upload/Studien/Gutachten/PDF/2018/Digital-Atlas.pdf (16.12.2019); Destatis (2019).

Abbildung 4 setzt branchenspezifische Arbeitsproduktivität ins Verhältnis zu dem von der IW Consult entwickelten Digital Index.11 Dieser verbindet in einer Vollerhebung aller deutschen Unternehmen Webcrawling-basierte Informationen von deren Website mit Unternehmensdaten und bildet so eine Mischung aus Digitisation und Digitalisation ab. Nicht nur zeigt sich im Branchenvergleich ein positiver Zusammenhang zwischen Digitalisierungsgrad und Produktivität, auch wird deutlich, dass die Digitalisierung in Deutschland im Verarbeitenden Gewerbe bereits im Jahr 2018 hinter der Informations- und Kommunikationstechnik-Branche (IKT-Branche) am zweitweitesten vorangeschritten ist. Bedeutsam ist dies, da Produktivitätssteigerungen im B2B-Markt besonders zu erwarten sind: Einerseits entstehen durch eine engmaschigere Echtzeitüberwachung des Produktionsprozesses Effizienzgewinne, andererseits lassen sich durch eine einfachere Produktdifferenzierung kleine Innovationen auch in geringen Tranchen auf den Markt bringen. So können Betreibern von Maschinen und Anlagen durch Vernetzung mit dem Hersteller vorausschauende Serviceangebote gemacht werden, Produktionsunterbrechungen werden verkürzt. Die zu erwartenden Auswirkungen auf die Produktivität sind hier erheblich, werden auf Makroebene aber erst

11 Vgl. IW Consult: Digital-Atlas Deutschland: Überblick über die Digitalisierung von Wirtschaft und Gesellschaft sowie von KMU, NGOs, Bildungseinrichtungen sowie der Zukunft der Arbeit in Deutschland, Köln 2018, https://www.iwkoeln.de/fileadmin/user_upload/Studien/ Gutachten/PDF/2018/Digital-Atlas.pdf (16.12.2019). 
sichtbar, wenn ausreichend viele Unternehmen Industrie-4.0-Technologien anwenden. ${ }^{12}$

Demgegenüber basieren Produktivitätssteigerungen im B2C-Markt weitestgehend auf der kostengünstigen Skalierbarkeit von Dienstleistungen, was ebenfalls Produktivitätssteigerungen auslösen dürfte. Dabei sind vor allem bessere Prozesse und unmittelbares Nachsteuern die treibende Kraft (Digital Transformation). An der C2BSchnittstelle ergeben sich nur bedingt Produktivitätssteigerungen. Verringerte Suchkosten durch nutzerspezifische Werbung bedeuten zwar einen Mehrwert für den Konsumenten, dieser tritt aber kaum in der gesamtwirtschaftlichen Produktivität zutage. Auch im C2C-Markt sind die Produktivitätszuwächse eher unerheblich. Lediglich die höhere Auslastung und der gesteigerte Wettbewerb der Plattformmärkte haben positive Produktivitätseffekte.

Die Diskrepanz zwischen den erwarteten Produktivitätseffekten und dem empirischen Befund eines stagnierenden oder rückläufigen Produktivitätswachstums lassen sich auf der Schnittstellen-Ebene durchaus auflösen. Ein vertiefender Blick in das Verarbeitende Gewerbe in Deutschland zeigt zudem, weshalb auch hier noch keine übermäßigen Produktivitätssprünge beobachtbar sind. So zeigt eine Umfrage des IW-Zukunftspanels zu den erwarteten Auswirkungen der Digitalisierung zwar mit Blick auf die eigene Wettbewerbsfähigkeit eine positive Erwartungshaltung der befragten Unternehmen, ${ }^{13}$ insbesondere bei der Frage nach datengetriebenen Geschäftsmodellen (der Aufbruch in die Digital Transformation über Digitisation) muss die breite Mehrheit der Unternehmen jedoch passen: Fast drei Viertel der deutschen Firmen gibt sogar an, Daten aktuell nicht zu bewerten und dies auch in Zukunft nicht zu planen. Folglich kommen Azkan et al. ${ }^{14}$ zu dem Schluss, dass in der Industrie beim Thema Data Economy ein Großteil der Unternehmen als „Einsteiger“ $(89,0 \%)$ oder Fortgeschrittene $(10,1 \%)$ zu klassifizieren sind. Der Anteil an „Pionieren“ liegt damit bei lediglich

12 M. Diermeier, H. Goecke: Productivity, Technology Diffusion and Digitalization, in: CESifo Forum, 18. Jg. (2017), H. 1, S. 26-32, https:// www.ifo.de/DocDL/CESifo-Forum-2017-1-diermeier-goecke-digitalization-march.pdf (27.2.2020).

13 Während $37 \%$ der befragten Unternehmen „positive“ oder „sehr positive" Auswirkungen der Digitalisierung auf die Wettbewerbsfähigkeit angeben, nennen lediglich $8 \%$ „negative“ oder „sehr negative“ Erwartungen. Vgl. IW-Zukunftspanel: 27. Welle 2016, Berlin 2019, https://www.wirtschaftsdienst.eu/files/veranstaltungen/2019-12-02-Digitalisierung/michael-huether_umsetzung-digitalisierung-unternehmensebene.pdf (17.12.2019).

14 Vgl. C. Azkan et al.: Bereitschaft der deutschen Unternehmen für die Teilhabe an der Datenwirtschaft, IW Köln 2019, https://www.iwkoeln. de/studien/gutachten/beitrag/vera-demary-manuel-fritsch-henrygoecke-alevtina-krotova-karl-lichtblau-edgar-schmitz-bereitschaftder-deutschen-unternehmen-fuer-die-teilhabe-an-der-datenwirtschaft.html (17.12.2019).
0,9\%, was sowohl relativ als auch absolut das noch unerfüllte Potenzial aufzeigt, das der digitale Wandel für viele Industrieunternehmen mit sich bringt - nämlich aus seinen analogen Strukturen heraus, digitale und datengetriebene Geschäftsmodelle zu entwickeln, die aufgrund der Kombination von Agilität und Skalierbarkeit letztlich auch ein großes Produktivitätswachstum mit sich bringen.

\section{Das große digitale Schreckgespenst: Beschäftigungsabbau}

Ähnlich kontrovers wie die Prognosen zur Produktivitätsentwicklung wird die Fragestellung diskutiert, wie sich der digitale Wandel auf das gesamtwirtschaftliche Beschäftigungsvolumen auswirken wird. Nachdem Projektionen mit dem potenziell vollständigen Verlust vieler Berufsbilder Schlagzeilen gemacht haben, ${ }^{15}$ prognostizierten tiefergreifende Analysen mit Blick auf Tätigkeitsprofile zuletzt geringere Effekte. ${ }^{16}$ Dieser Befund öffnet den Raum für ein wichtiges Argument: Auch wenn Digitalisierung nicht zwingend mit dem Wegfall von ganzen Berufen und Arbeitsplätzen einhergeht, könnten einzelne, standardisierbare, monotone Arbeitsschritte durchaus rationalisiert werden (Digitisation). Auch bei der Implementierung von „Arbeit ohne Arbeiter“ in der Industrie - also einer rein Maschinen-basierten Produktion - werden zwar Effizienzgewinne erzielt, es kann aber nicht alles maschinell abgebildet werden, was Arbeitnehmer vorher abgedeckt haben. Denn neben seinen standardisierbaren Aufgaben stellt der Mensch noch immer eine wichtige Prüfinstanz dar, die beispielsweise Fehler aus vorherigen Arbeitsschritten ausmachen und beheben kann. Automatisierte Systeme und auch künstliche Intelligenz (KI) sind nicht oder nur eingeschränkt dazu fähig, sich auf verändernde Umgebungen und Anforderungen einzulassen. So hat selbst Elon Musk nach der Implementierung einer im Nachhinein als übermäßig identifizierten Automatisierung in seinen Tesla-Werken anmerken müssen: „Menschen werden unterschätzt"17.

Bei der genaueren Einordnung der Beschäftigungseffekte lohnt es sich abermals, die Perspektive der vier Produzenten-Kundenbeziehung-Schnittstellen einzunehmen:

15 Vgl. C. Osborne, M. Frey: The future of employment: How susceptible are jobs to computerisation?, Oxford Martin Programme on Technology and Employment 2013, https://www.oxfordmartin.ox.ac.uk/downloads/academic/The_Future_of_Employment.pdf (17.12.2019).

16 Vgl. H. Bonin, T. Gregory, U. Zierahn: Übertragung der Studie von Frey/Osborne (2013) auf Deutschland: Kurzexpertise, Nr. 57, Zentrum für Europäische Wirtschaftsforschung (ZEW), Mannheim 2015 http://ftp.zew.de/pub/zew-docs/gutachten/Kurzexpertise_BMAS_ ZEW2015.pdf (17.12.2019).

17 Vgl. E. Musk: Yes, excessive automation at Tesla was a mistake. To be precise, my mistake. Humans are underrated [Twitter Post], 13.4.2018, https://twitter.com/elonmusk/status/98488263094775398 4 ?lang=de $(17.12 .2019)$. 
Positive Beschäftigungseffekte sind mittelfristig insbesondere im Business-to-Business-Markt zu erwarten. Hier ist implizites, in Fachkräften gebundenes Fachwissen, noch immer nicht durch Maschinen und Algorithmen zu substituieren - freilich mögen langfristig KI-basierte Algorithmen ganze Berufsgruppen auch gänzlich ablösen, ein solches Szenario liegt jedoch in weiter Ferne. Die C2B-Schnittstelle ist kaum mit Beschäftigung unterlegt, sodass negative Beschäftigungseffekte praktisch ausgeschlossen sind. Erstaunlicherweise sind auch in der B2CSchnittstelle bislang keine größeren Beschäftigungseffekte auf der Makroebene aufgetreten. Die drei prominentesten Tech-Unternehmen Facebook, Google und Apple kommen zusammen weltweit auf lediglich rund 295000 Vollzeitbeschäftigte ${ }^{18}$ - verglichen mit klassischen Industrieunternehmen ist das recht wenig.

Im C2C-Markt wird die Digitalisierung voraussichtlich leicht negativ auf das gemessene Beschäftigungsvolumen wirken - wohingegen die Effekte auf die tatsächliche Beschäftigungsentwicklung dabei durchaus neutral bleiben könnten. Dies wäre etwa der Fall, wenn Plattform-basierte Fahrdienste klassische Taxiunternehmen aus dem Markt drängen und ihre Arbeitszeit nicht gänzlich gewerblich erfasst würde. Darüber hinaus ist in diesem Bereich ein Wandel hin zu dezentral organisierter Beschäftigung bemerkbar. Insgesamt bleibt der Arbeitsmarkt rund um die Schnittstelle C2C am schwierigsten zu beurteilen, da hier am ehesten größere Messfehler auftreten können. Die Bedeutung gegenüber den anderen Schnittstellen wie dem B2B-Markt ist aufgrund der geringen Beschäftigungshinterlegung jedoch als gering einzuschätzen.

Für eine differenzierte Aussage aus Branchenperspektive lohnt sich der Blick auf die Unternehmensangaben zum Beschäftigungsausblick. Hier zeigen sich deutliche Unterschiede in den Beschäftigungsprognosen von Unternehmen der unterschiedlichen Wirtschaftsbereiche. Während in der Finanzwirtschaft $61 \%$ der Unternehmen von einer Beschäftigungsreduzierung ausgehen, sind es im Bau- und Gastgewerbe sowie im IKT-Sektor lediglich $11 \%$ bzw. $12 \%$. In der Industrie überwiegen die negativen Erwartungen leicht (vgl. Abbildung 5). Auch wenn in diesem Ausblick der digitale Wandel nur implizit mitschwingt, zeigt sich doch: Mit Ausnahme der Finanzindustrie erwartet kaum eine Branche einen klaren Trend zum Beschäftigungsabbau.

18 Vgl. Alphabet Inc.: Alphabet Announces Third Quarter 2019 Results, Mountain View CA 2019, https://abc.xyz/investor/static/pdf/2019Q3 alphabet_earnings_release.pdf?cache $=\mathrm{d} 41 \mathrm{c} 776$ (17.12.2019); Facebook: Our Offices, Menlo Park CA 2019, https://about.fb.com/company-info/ (17.12.2019); Apple Inc.: Annual Report, Cupertino California 2019, https://s2.q4cdn.com/470004039/files/doc_financials/2019/ ar/_10-K-2019-(As-Filed).pdf (17.12.2019).

\section{Abbildung 5}

Ambivalente Aussichten für Beschäftigungsperspektiven

„Welche Auswirkungen hat die Digitalisierung in Ihrem Unternehmen?", Anteil der befragten Unternehmen, in \%

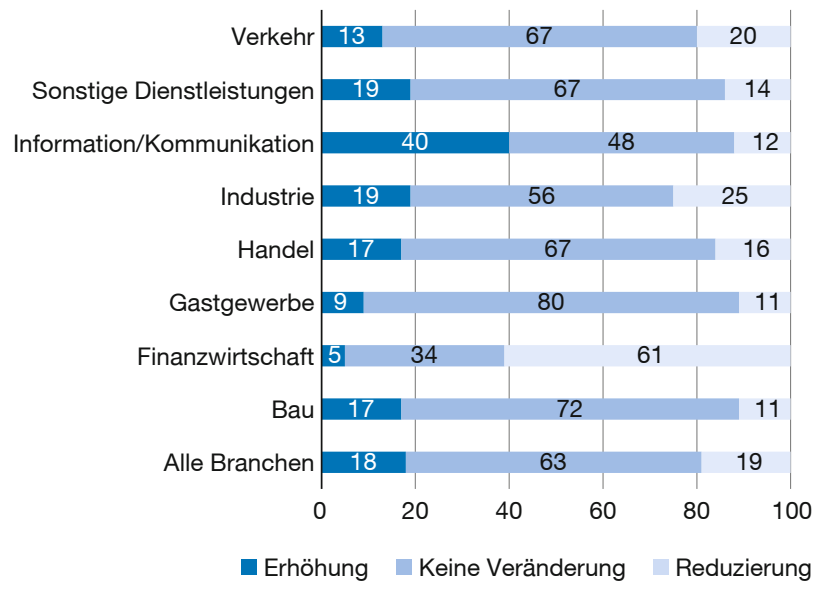

Quelle: Deutscher Industrie- und Handelskammertag: Wachsende Herausforderungen treffen auf größeren Optimismus - Das IHK-Unternehmensbarometer zur Digitalisierung, Berlin 2017.

Doch dies bedeutet mitnichten, dass es nicht zu einer deutlichen Veränderung der Kompetenzanforderungen kommen wird. Die Differenzierung der Beschäftigungseffekte nach Kompetenzanforderungen zeigt auf der Mikroebene eine merkliche Strukturverschiebung an. So werden besonders Arbeitskräfte mit IT-Fachwissen, Planungs- sowie Kommunikationsfähigkeit gesucht und benötigt. ${ }^{19}$ Interessanterweise stehen sehr spezifische IT-Fachkenntnisse zu Softwareprogrammierung oder Industrie-4.0-Technologien nicht am obersten Ende der Anforderungen in den Unternehmen. Auch hier zeigt sich wieder, dass die Unternehmen eher einen sukzessiven als einen disruptiven Wandel erwarten: Nicht alle Fachkräfte werden in Zukunft zu IT-Spezialisten umgeschult, aber alle Mitarbeitenden werden gewisse IT-Anwendungsfähigkeiten vorweisen müssen. Bei den großen Tech-Konzernen im B2C-Bereich sowie einigen unternehmensnahen Dienstleistern, die Industrie-4.0-Technologien betreuen, mag dies freilich anders aussehen, für die durchschnittlichen Arbeitnehmer in der deutschen Wirtschaft ergibt sich jedoch eine zwar drängende, aber doch eher sukzessive als disruptive Veränderung des Anforderungsprofils.

19 Vgl. M. Wolter et al.: Wirtschaft 4.0 und die Folgen für Arbeitsmarkt und Ökonomie: Szenario-Rechnungen im Rahmen der BIBB-IABQualifikations- und Berufsfeldprojektionen, in: IAB Forschungsbericht (2016), H. 13, http://doku.iab.de/forschungsbericht/2016/fb1316. pdf (17.12.2019). 


\section{Abbildung 6}

Entwicklung der Bedeutung von Kompetenzen im Zuge der Digitalisierung

Anteil der Unternehmen, die für die folgenden Fähigkeiten in den kommenden fünf Jahren von einem Bedeutungszuwachs ausgehen, 2018

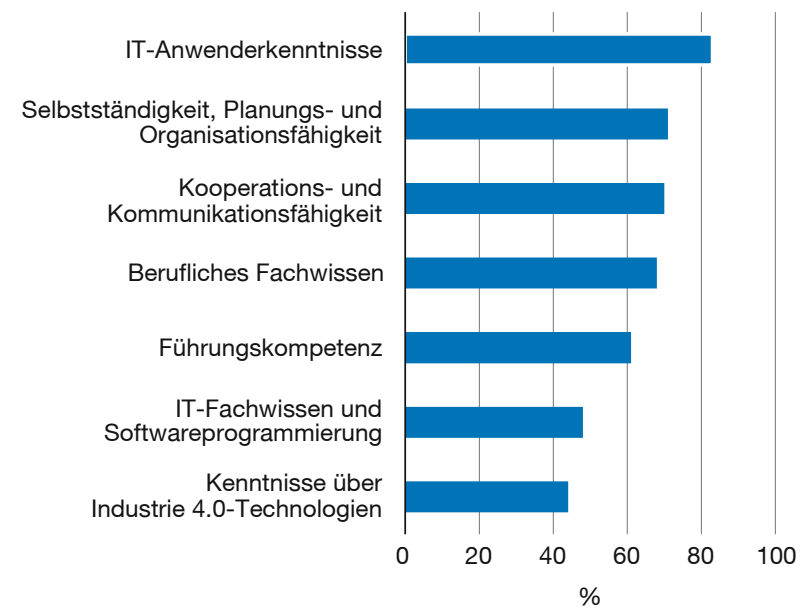

Quelle: Kompetenzzentrum Fachkräftesicherung: Digitale Bildung in Unternehmen - Wie KMU E-Learning nutzen und welche Unterstützung sie brauchen, Köln 2019.

Nichtsdestotrotz zeigt sich, dass Unternehmen mehr denn je in der Verantwortung stehen, ihren Mitarbeitenden die Chance zu geben, sich zukunftsträchtige IT-Anwenderkenntnisse anzueignen (vgl. Abbildung 6). Insbesondere im Kontext des demografischen Wandels erreicht das lebenslange Lernen einen neuen Imperativ. Zum ersten Mal in der Geschichte der großen technologischen Umwälzungen müssen die Veränderungen auch von den älteren Generationen von Arbeitnehmern breit mitgetragen werden. Welche Herausforderung dies bergen wird, zeigt ein kurzer Blick auf digitale Fähigkeiten in den unterschiedlichen Alterskohorten. Während $62 \%$ der 16- bis 24-Jährigen über fortgeschrittene digitale Fähigkeiten verfügen, beläuft sich dieser Anteil bei den 25- bis 54-Jährigen lediglich auf $43 \%$ und bei den 55 bis 74 -Jährigen sogar nur auf $16 \%{ }^{20}$ Schon heute zeigen sich regionalspezifisch die entsprechenden Auswirkungen auf die Produktivitätsentwicklung: Kreise mit einem höheren Beschäftigungsanteil der über 55-Jährigen haben während der vergangenen 15 Jahre ein geringeres Produktivitätswachstum aufgewiesen. ${ }^{21}$

20 Vgl. Eurostat: Individuals‘ level of digital skills, 2017, http://appsso.eurostat.ec.europa.eu/nui/show.do?dataset=isoc_sk_dskl_i\&lang=en (17.12.2019).

21 Vgl. Sachverständigenrat zur Begutachtung der gesamtwirtschaftlichen Entwicklung: Den Strukturwandel meistern: Jahresgutachten 2019/20, Wiesbaden 2019, Zf. 203.

\section{Die digitale Transformation vorantreiben}

Wie gut ein Unternehmen auf die anstehenden Umbrüche vorbereitet ist, hängt maßgeblich von seiner Fähigkeit ab, sich auf Strukturveränderungen einzustellen und innovative Antworten auf sich wandelnde Nachfrage- und Konkurrenzmuster zu entwickeln. Kempermann und Pohl22 zeigen in einer mehrstufigen Clusteranalyse eindrücklich die Spaltung, die sich diesbezüglich durch die deutsche Unternehmenslandschaft zieht. Während Unternehmen in Innovations-affinen Milieus ein hohes Produktivitätswachstum aufweisen und die Digitalisierung ihrer Prozesse (Digitisation) und zum Teil sogar ihrer Geschäftsmodelle (Digitalisation) vorantreiben, fallen Unternehmen in Innovations-fernen Milieus Stück für Stück zurück. Vermutlich wird sich dieser Trend in Zukunft noch verschärfen, denn Unternehmen im digitalaffinen Milieu betreiben einen erheblichen Aufwand, um ihre gute Wettbewerbsposition zu sichern: Während im Spitzenmilieu rund 5,8\% des Gesamtumsatzes in die "digitale Transformation" investiert wird, liegt dieser Anteil in Unternehmen ohne Innovationsfokus bei lediglich $1,7 \%$.

Deutlich zeigt sich zudem eine geografische Spaltung zwischen der digitalen Avantgarde und den Nachzüglern. Der Digital Index der IW Consult verdeutlicht, wie insbesondere kleinere Unternehmen, die auf dem Land angesiedelt sind, zunehmend ins Hintertreffen geraten. Unternehmen in (westdeutschen) Groß- und Kernstädten haben deutlich die Nase vorn. Je weniger urban die Siedlungsstruktur in einer Region ausfällt, desto geringer fällt der Digitalisierungsgrad der ansässigen Unternehmen aus. ${ }^{23} \mathrm{Zu}$ einem ähnlichen Befund kommt eine Regionalanalyse der Patentanmeldungen mit Digitalisierungsbezug: Während in München deutschlandweit jedes vierte solche Patent angemeldet wird, gibt es in mehr als jedem dritten Landkreis überhaupt keine Patenanmeldung mit Bezug zu einer Digitalisierungstechnologie. ${ }^{24}$

Dass einige Unternehmen innovativer und erfolgreicher sind als andere und sich bestimmte Cluster in einem Strukturwandel besser behaupten können, ist bis zu einem gewissen Grad wenig verwunderlich. Problematisch wird eine solche Entwicklung erst dann, wenn Rahmenbedingungen asymmetrisch den Gestaltungsspielraum von Unternehmen oder Arbeitnehmern beeinflussen - wenn

22 Vgl. H. Kempermann, P. Pohl: Innovative Milieus in Deutschland, in: IW-Trends, 46. Jg. (2019), H. 3, S. 91-108, https://www.iwkoeln.de/fileadmin/user_upload/Studien/IW-Trends/PDF/2019/IWTrends_2019-03-06_Innovative_Milieus_in_D.pdf (17.12.2019).

23 Vgl. IW Consult: Digital-Atlas Deutschland, a.a. O., S. 109.

24 S. Berger, O. Koppel, E. Röbben: Deutschlands Hochburgen der Digitalisierung, IW-Kurzberichte, Nr. 42, 2017, https://www.iwkoeln.de/ fileadmin/publikationen/2017/340150/IW-Kurzbericht_42_2017_Digitalisierungspatente.pdf (17.12.2019). 
also manchen Akteuren von vornherein die Möglichkeit genommen wird, sich in ihrer Lebenswirklichkeit am fairen Wettbewerb um die besten Ideen zu beteiligen. Aus dieser Überlegung ergeben sich vier konkrete Ableitungen:

- Im Kontext des demografischen Wandels ist es unabdingbar, insbesondere älteren Arbeitnehmern im B2BBereich stetig passgenaue Weiterbildungsangebote zu bieten. Lebenslanges Lernen ist die einzige valide Antwort auf einen Strukturwandel, der zum Großteil innerhalb einer einzigen Kohorte von Arbeitnehmern bewältigt werden muss. ${ }^{25}$

- Auf regionaler Ebene ist die dezentral organisierte deutsche Wirtschaft darauf angewiesen, dass erfolgreiches Wirtschaften im ländlichen Raum möglich ist. Mangelhafte Infrastrukturversorgung sowie grassierender Fachkräftemangel stellen die regionale Chancengleichheit derzeit infrage. In besonderem Maße gilt dies für Ostdeutschland. ${ }^{26}$

- Wettbewerbspolitisch stellt die Digitalisierung neue Fragen, da einerseits der Zugang zu und die Bewirtschaftung der Daten mit erheblichen ökonomischen Potenzialen der Marktbeeinflussung und Marktdominanz verbunden sind, andererseits Plattformen als Intermediäre das bekannte Phänomen zwei- oder mehrseitiger Märkte infolge direkter und indirekter Netzwerkeffekte qualitativ neu positionieren (Potenzial

25 Vgl. S. Schöpper-Grabe, I. Vahlhaus: Grundbildung und Weiterbildung für Geringqualifizierte, in: IW-Trends, 46. Jg. (2019), H. 1, S. 45-60, https://www.iwkoeln.de/fileadmin/user_upload/Studien/IW-Trends/ PDF/2019/IW-Trends_2019-01-03_Grundbildung_Geringqualifizierte_.pdf (17.12.2019).

26 Vgl. H. Kempermann, A. Millack: Digitale Regionen in Deutschland, in: IW-Trends, 45. Jg. (2018), H. 1, S. 49-66, https://www.iwkoeln.de/ fileadmin/publikationen/2018/382376/IW-Trends_2018-03_Digitale_Regionen.pdf (17.12.2019). zum Marktverschluss). Nachdem mit der 9. Novelle des Gesetzes gegen Wettbewerbsbeschränkungen (GWB) 2017 erste Schritte gemacht wurden, steht nun die 10. GWB-Novelle (Multihoming, „Intermediationsmacht“, Datenzugang, besondere Verantwortung von Plattformen) in der Diskussion. ${ }^{27}$ Deutschland (und Europa) haben hier die Chance, wichtige Orientierung weltweit zu geben.

- Zudem muss im globalen Wettbewerb um Daten allen Akteuren klar sein, nach welchen Regeln gespielt wird. Auf der einen Seite bedeutet dies ein verständliches Vermitteln, wofür Kundendaten gespeichert und genutzt werden. Auf der anderen Seite bedeutet dies auch, dass europäische Unternehmen gegenüber ihren chinesischen und amerikanischen Counterparts keinen Nachteil haben dürfen. Eigentumsrechte müssen klar zugewiesen werden. ${ }^{28}$

- Hier schließt sich die Frage nach globalen Standards zu Datentransfers an. Eine fehlende Standardisierung schafft Friktionen und Kosten, eine gemeinsame Sprache ist unabdingbar für reibungslose Abläufe insbesondere im Lichte von Industrie-4.0-Technologien. Welches Unternehmen, aber auch welcher Wirtschaftsraum es schafft, seine Standards global attraktiv zu machen, wird dem Wirtschaften im digitalen Zeitalter seinen Fußabdruck aufdrücken. ${ }^{29}$

27 Vgl. dazu den Beitrag von J. Haucap: Plattformökonomie: neue Wettbewerbsregeln - Renaissance der Missbrauchsaufsicht, in: Wirtschaftsdienst, 100. Jg. (2020), H. 13, S. 20-29.

28 Vgl. C. Azkan et al., a.a. O.

29 Vgl. B. Engels: Bedeutung von Standards für die digitale Transformation, in: IW-Trends, 44. Jg. (2017), H. 2, S. 21-40, https://www.iwkoeln de/fileadmin/publikationen/2017/339509/IW-Trends_2017-02_Standards_Digitalisierung.pdf (17.12.2019).

Title: Potentials and implementation of digitisation at company level

Abstract: Digitisation at the corporate level has long been a practical component of strategic planning and implementation. Specific effects on productivity growth or employment can be shown on the basis of producer-customer relationships. With regard to productivity growth, it is to be expected that increases will be delayed and that there is still untapped potential in many areas. Positive employment effects can be expected especially in the business-to-business market, while the business-to-consumer market, which is dominated by the large tech companies, has comparatively low employment figures. The bottom line is that there will increasingly be a change in the competence requirements. The challenge is not to lose out on less innovation-affine companies and fewer IT-affine employees.

JEL Classification: LO, L2, O3 